\title{
Risk factors associated with moderate-to-severe renal dysfunction among heart transplant patients: results from the CAPRI study
}

\author{
Juan F. Delgado, María G .Crespo-Leiro, Miguel A. Gómez-Sánchez, María J. \\ Paniagua, Francisco González-Vílchez, José A.Vázquez de Prada, Juan Fernández- \\ Yáñez, David Pascual, Luis Almenar, Luis Martínez-Dolz, Beatriz Díaz, Eulalia Roig, \\ Javier Segovia, Jose M. Arizón, Iris Garrido, Teresa Blasco, Javier López, Vicens \\ Brossam, Nicolás Manito and Javier Muñiz
}

\begin{abstract}
The longer survival of patients with heart transplantation (HT) favors calcineurin inhibitor-related chronic kidney disease (CKD). It behoves to identify risk factors. At 14 Spanish centers, data on 1062 adult patients with HT (age $59.2 \pm 12.3 \mathrm{yr}, 82.5 \%$ men) were collected at routine follow-up examinations. Glomerular filtration rate, GFR, was estimated using the four-variable MDRD equation, and moderate-or-severe renal dysfunction (MSRD) was defined as $\mathrm{K} / \mathrm{DOQI}$ stage $3 \mathrm{CKD}$ or worse. Time since transplant ranged from one month to $22 \mathrm{yr}$ (mean $6.7 \mathrm{yr}$ ). At assessment, $26.6 \%$ of patients were diabetic and $63.9 \%$ hypertensive; $53.9 \%$ were taking cyclosporine and $33.1 \%$ tacrolimus; and $61.4 \%$ had MSRD. Among patients on cyclosporine or tacrolimus at assessment, multivariate logistic regression identified male sex (OR 0.44), pre- and post-HT creatinine (2.73 and 3.13 per mg/dL), age at transplant (1.06 per yr), time since transplant (1.05 per yr), and tacrolimus (0.65) as independent positive or negative predictors of MSRD. It is concluded that female sex, pre- and one-month post-HT serum creatinine, age at transplant, time since transplant, and immunosuppression with cyclosporine rather than tacrolimus may all be risk factors for development of $\mathrm{CKD} \geq$ stage 3 by patients with HT.
\end{abstract}

The life expectancy of patients with heart transplantation (HT) is steadily increasing. According to the ISHLT Registry report for 2008, the estimated median survival time among patients surviving the first yr after transplantation, calculated from data for 1982-2006, is $13 \mathrm{yr}$ (1). Longer survival brings with it a greater risk of non-cardiac morbidities because of normal aging (such as diabetes), immunosuppression (such as neoplasia and infections), and adverse side effects of immunosuppressive therapy that do not derive directly from immunosuppression per se. In particular, the nephrotoxic effects of calcineurin inhibitors (CNI) have been of concern virtually since the introduction of cyclosporine (2), both on their own account and because of the cardiovascular and other non-renal pathological implications of renal dysfunction (3). Post-transplant chronic kidney disease (CKD) of K/DOQI (4) stage 4 or worse multiplies four-fold the death rate among all non-renal solid organ transplant patients (5), and the five-yr survival of patients with HT on dialysis for CKD is only half that of dialyzed patients with CKD with no organ graft (19\% as against 40\%) (6). The ISHLT-Registry-based Kaplan-Meier estimate of the intrinsic probability of an patient with HT who survives $13 \mathrm{yr}$ developing severe renal dysfunction (serum creatinine $>2.5 \mathrm{mg} / \mathrm{dL}$, dialysis or kidney transplant) exceeds $40 \%$ (1); the current actual prevalence of CKD of $\mathrm{K} / \mathrm{DOQI}$ stage 3 or worse among Spanish patients with HT who survive more than $9.5 \mathrm{yr}$ is $67 \%$ (7).

The magnitude of the problem of post-transplant CKD makes it necessary to characterize patients at greatest risk, if only because this will facilitate preliminary evaluation of preventive strategies (8). Most studies that have investigated this issue have identified risk factors analogous to those of CKD of nontransplant patients, including age, female sex, pre-transplant arterial hypertension and diabetes mellitus, together with pre-transplant renal dysfunction and post-operative acute kidney failure $(3,5)$. There has been less concord at least for patients with HT - regarding other possible risk factors, including serum triglycerides, original ischemic cardiopathy, blood urea and alkaline phosphatase, weight, body mass index, race, donor hypertension, and pre-transplant hepatitis $\mathrm{C}$ infection $(1,9-12)$.

Here, we report risk factors for K/DOQI stage 3 CKD or worse that were identified by CAPRI, a cross-sectional observational study carried out in 14 Spanish heart transplant centers in late 2007 and early 2008 with the primary objective of determining the current prevalence of kidney dysfunction among Spanish patients with HT and risk factors therefore. 


\section{Patients and methods}

\section{Participants}

Between October 2007 and March 2008, each of 14 Spanish heart transplant centers fed the CAPRI database with relevant data on all first patients with HT aged $>18$ who presented during a pre-defined three-month period for a routine follow-up examination more than $30 \mathrm{~d}$ post-transplant (informed consent was a further condition that gave rise to no exclusions). Data were recorded for a total of 1062 patients (82.5\% males) with a mean age of $59.2 \pm 12.3 \mathrm{yr}$ and times since transplant ranging from one month to $22.3 \mathrm{yr}$ (mean $6.7 \mathrm{yr}$, SD $4.6 \mathrm{yr}$ ). Before transplant, $15.0 \%$ had received a diagnosis of diabetes mellitus (cf. $26.5 \%$ at the time of the study), $29.8 \%$ were hypertensive (cf. $64.1 \%$ at study time), and $2.0 \%$ were seropositive for hepatitis C. The original cardiopathy leading to HT was dilated cardiomyopathy in $40.6 \%$, and ischemic cardiomyopathy in $39.8 \%$. Some $43.4 \%$ had suffered an episode of acute rejection of ISHLT grade 3A or worse in the first yr after transplant, and at the time of the study, $48.9 \%$ had been treated for cytomegalovirus infection. Table 1 lists details of immunosuppression at the time of the study.

Table 1. Immunosuppressive medication of 1062 Spanish patients with heart transplantation (HT)

\begin{tabular}{|c|c|c|c|}
\hline Immunosuppressant & & Prevalence $(\%)$ & \\
\hline Calcineurin inhibitor & & & 87.0 \\
\hline Cyclosporine & & 53.9 & \\
\hline Tacrolimus & & 33.1 & \\
\hline Antimetabolite & & & 81.3 \\
\hline Azathioprine & & 11.7 & \\
\hline Mycophenolate & & 69.6 & \\
\hline Mycophenolate mofetil & 66.7 & & \\
\hline Sodium mycophenolate & 2.9 & & \\
\hline Steroids & & & 68.3 \\
\hline mTOR inhibitor & & & 20.2 \\
\hline Sirolimus & & 5.9 & \\
\hline Everolimus & & 14.3 & \\
\hline
\end{tabular}

\section{Outcome variable}

The outcome variable was K/DOQI stage $3 \mathrm{CKD}$ or worse, hereinafter referred to for brevity as MSRD (moderate or severe renal dysfunction), and defined by the patients having an estimated glomerular filtration rate (eGFR) less than $60 \mathrm{~mL} / \mathrm{min}$ per $1.73 \mathrm{~m}^{2}$, or being on dialysis, or having undergone kidney transplant for post-HT kidney failure (4).

Glomerular filtration rate was estimated using the four-variable MDRD equation: eGFR $(\mathrm{mL} / \mathrm{min}$ per $\left.1.73 \mathrm{~m}^{2}\right)=186 \times \mathrm{SCr}^{-1.154} \times \mathrm{Age}^{-0.203} \times \mathrm{Sex} \times \mathrm{Race}$; where $\mathrm{SCr}$ is serum creatinine in $\mathrm{mg} / \mathrm{dL}$, Age is in $\mathrm{yr}$, Sex is 0.742 if female and 1 if male, and Race is 1.21 if black and 1 otherwise (4).

\section{Statistical analysis}

Patients with and without MSRD at the time of the study were compared with respect to relevant categorical variables by means of chi-squared tests, and with respect to continuous variables using Student's $t$-tests following verification of distributional normality by Kolmogorov-Smirnov tests. Independent predictors of MSRD at the time of the study were identified by means of backward stepwise multivariate logistic regression analysis of the data for the 977 patients for whom data were available for all the eight variables initially included in the model, to wit, all except two of the non-medicational variables for which significant between-group differences had been detected in the univariate analyses (age at transplant; pre-transplant diabetes, hypertension, and serum creatinine; serum creatinine $30 \mathrm{~d}$ after transplant; and time since transplant - hypertension and diabetes at study time were omitted as redundant) plus sex and the occurrence of acute rejection of ISHLT grade $\geq 3 \mathrm{~A}$ in the first yr post-transplant (these last two being included on clinical grounds; see the Discussion). Variables were dropped from the model if $\mathrm{p}<0.1$. To investigate the possible influence of tacrolimus (Tac) as against cyclosporine (CsA), two 
further multivariate logistic regressions were also performed on subsets of the 977, one including only the 924 patients started on CsA or Tac after transplantation, and the other only the 853 patients taking CsA or Tac at the time of the study. The variables included in these two regressions were seven that emerged as significant or near significant in the analysis of 977 patient (see Results; these seven were forced to remain in the model as possible confounders) plus either Tac versus CsA after transplantation, or Tac versus CsA at the time of the study. All statistical calculations were performed using SPSS for Windows v. 16.0. The criterion for statistical significance was $\mathrm{p}<0.05$.

\section{Results}

The prevalence of MSRD in the study group at the time of the study was $61.4 \%(652 / 1062)$. Among the pre-transplant characteristics examined, the MSRD and non-MSRD groups differed significantly with respect to age at transplant (MSRD $55.5 \pm 9.9 \mathrm{yr}$, non-MSRD $47.8 \pm 13.3 \mathrm{yr} ; \mathrm{p}<0.001$ ), serum creatinine $(1.35 \pm 0.63$ vs. $1.13 \pm 0.47 \mathrm{mg} / \mathrm{dL} ; \mathrm{p}<0.001)$, diabetes $(17.9 \%$ vs. $10.2 \% ; \mathrm{p}=0.001)$, and arterial hypertension $(34.5 \%$ vs. $22.2 \%$; p $<0.001)$, but not with respect to sex or the original cardiopathy that had led to HT (Table 2). Among other variables of potential utility for prediction of MSRD, the MSRD and non-MSRD groups differed significantly with respect to serum creatinine $30 \mathrm{~d}$ after transplant (MSRD $1.35 \pm 0.73$, non-MSRD $1.06 \pm 0.38 ; \mathrm{p}<0.001$ ) and the time elapsed between transplant and study $(7.1 \pm 4.7$ vs. $6.0 \pm 4.4 \mathrm{yr} ; \mathrm{p}<0.001)$, but not with respect to seropositivity for hepatitis $\mathrm{C}$ virus, or treatment for cytomegalovirus infection, or the incidence of acute rejection $\geq$ ISHLT grade $3 \mathrm{~A}$ in the first yr after transplant (Table 2).

Table 2. Comparisons with respect to non-medication variables between patients with heart transplantation (HT) with and without moderate or severe renal dysfunction (MSRD) at the time of the study

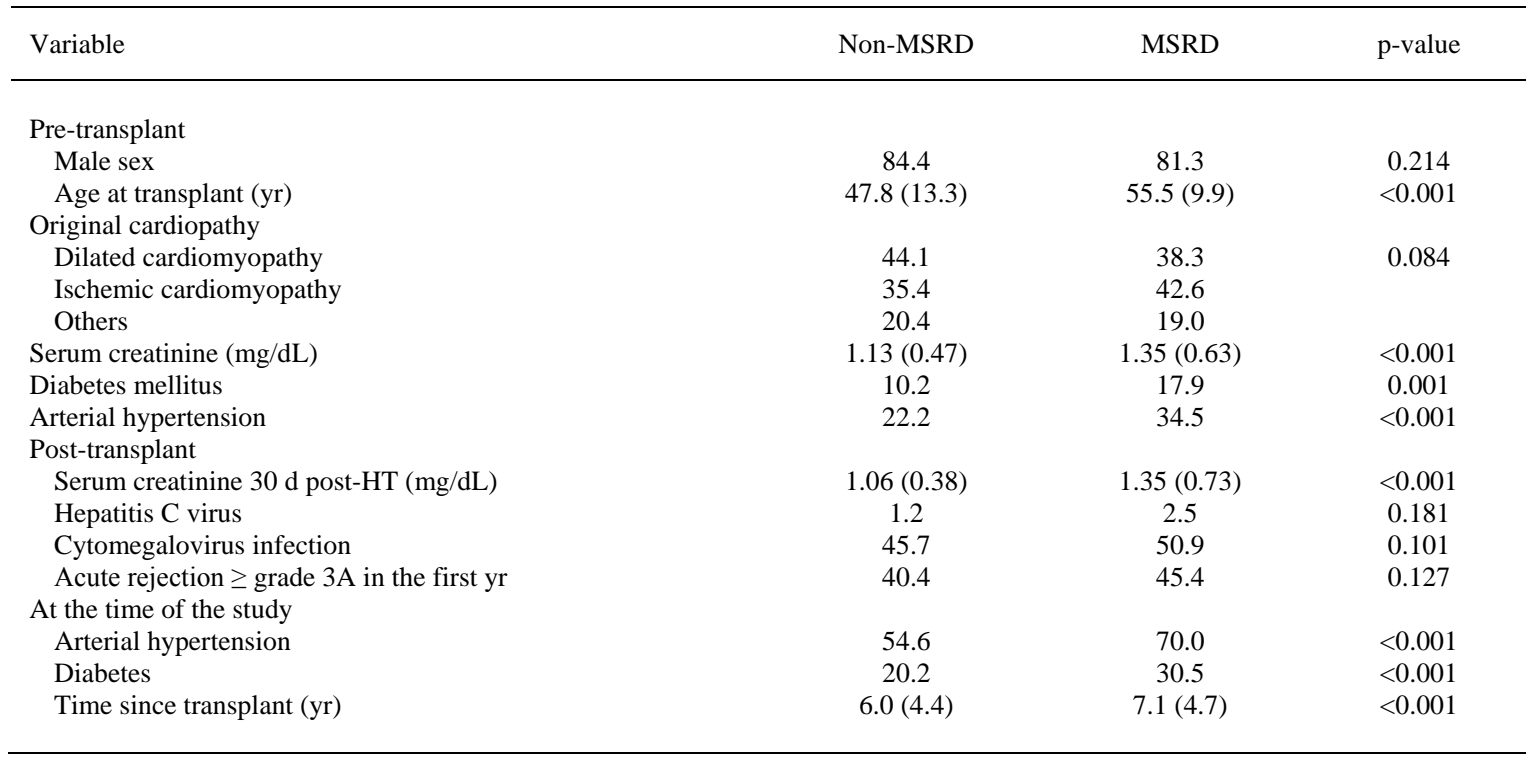

Data are percentages, or means with standard deviations in parentheses.

In both groups, the prevalences of both arterial hypertension and diabetes at the time of the study were about double what they had been before transplant, increasing the difference between the two groups with respect to these variables.

There were also significant differences between the MSRD and non-MSRD groups as regards their immunosuppressive regimens, both at initiation of immunosuppression and at the time of the study; in particular, at both times, smaller proportions of the MSRD group received Tac (Table 3). Additionally, larger proportions of the MSRD group were started on CsA and azathioprine and a smaller proportion on mycophenolate; and at study time, the prevalence of immunosuppression with sirolimus or everolimus was greater in the MSRD group, and the prevalence of azathioprine was smaller (Table 3). 
Table 3. Comparisons between patients with heart transplantation (HT) with and without MSRD at the time of the study with respect to immunosuppressive medication used for initiation of immunosuppression, and immunosuppressive medication at the time of the study

\begin{tabular}{|c|c|c|c|}
\hline Variable & Non-MSRD & MSRD & $\mathrm{p}$ \\
\hline \multicolumn{4}{|l|}{ At initiation } \\
\hline Steroids & 95.9 & 97.2 & 0.222 \\
\hline Cyclosporine & 75.6 & 80.8 & 0.045 \\
\hline Tacrolimus & 19.5 & 13.5 & 0.01 \\
\hline Sirolimus & 1.7 & 1.7 & 1 \\
\hline Everolimus & 2.2 & 3.1 & 0.445 \\
\hline \multicolumn{4}{|l|}{ At study time } \\
\hline Steroids & 65.4 & 70.1 & 0.119 \\
\hline Cyclosporine & 51.7 & 55.2 & 0.283 \\
\hline Everolimus & 11.0 & 16.4 & 0.015 \\
\hline
\end{tabular}

Data are given in percentages.

The significant independent predictors of MSRD identified in the initial multivariate logistic regression analysis of data for 977 patients (see Patients and methods) were female sex, age at transplant, time since transplant, and serum creatinine before and $30 \mathrm{~d}$ after transplant $(\mathrm{p} \leq 0.001$ in all cases; odds ratios (ORs), and their 95\% confidence intervals (CIs) are listed in Table 4). Additionally, pre-transplant diabetes (OR 1.49, 95\% CI 0.97-2.29; $\mathrm{p}=0.066)$ and acute rejection $\geq$ grade $3 \mathrm{~A}$ in the first $\mathrm{yr}(\mathrm{OR} 1.31$, $95 \%$ CI $0.98-1.77 ; \mathrm{p}=0.072$ ) came near to statistical significance. When all these variables were adjusted in the multivariate analysis that included the variable Tac vs. CsA at institution of immunosuppression, Tac vs. CsA was not significant (OR 0.755, 95\% CI 0.501-1.140; $\mathrm{p}=0.182$ ). However, in the analysis of data for 853 patients taking CsA or Tac at the time of the study, Tac vs. CsA emerged as a significant independent predictor alongside all the other variables except pre-transplant diabetes and acute rejection, Tac being less associated with MSRD than CsA (OR 0.65, 95\% CI 0.46$0.90 ; \mathrm{p}=0.011$.

Table 4. Variables with significant or near-significant association with MSRD in a multivariate logistic regression in which the initial variables were those shown here plus pre-transplant hypertension

\begin{tabular}{lcc}
\hline Variable & OR & $95 \%$ CI \\
\hline & & p value \\
Male sex & 0.519 & $0.346-0.777$ \\
Pre-transplant serum creatinine (per mg/dL) & 2.022 & $1.375-2.972$ \\
Age at transplant (per yr) & 1.060 & $1.046-1.074$ \\
Serum creatinine 30 d post-HT (per mg/dL) & 4.025 & $2.588-6.260$ \\
Time since transplant (per yr) & 1.077 & $1.043-1.113$ \\
Pre-transplant diabetes & 1.494 & $0.974-2.292$ \\
Acute rejection $\geq$ grade 3A in the first yr & 1.314 & $0.976-1.768$ \\
\end{tabular}

OR, odds ratio, $\mathrm{CI}$, confidence interval.

The analysis included data for the 977 patients for whom data were available for all eight variables. 
Suspecting, in view of the general evolution of immunosuppressive regimens, that this last result might be because of early patients with HT having received larger dosages of cyclosporine than later patients, we repeated this analysis including only the 633 patients treated after the introduction of tacrolimus 9 yrs before the study date, but the only noteworthy change in the results was that time since transplant was no longer a significant predictor; in particular, the influence of Tac vs. CsA was almost unaltered (OR 0.68, 95\% CI 0.47-0.98; $\mathrm{p}=0.038$ ).

\section{Discussion}

The risk factors for post-HT MSRD identified in this work were female sex, age at transplant, time since transplant, serum creatinine before and $30 \mathrm{~d}$ after transplant, and current maintenance on CsA rather than Tac. These factors mostly coincide with those identified in other studies of renal dysfunction among patients with HT and other non-renal solid organ transplant patients.

The variables of the initial models employed in the regression analyses from which these predictors emerged included sex and acute rejection in the first yr, in spite of these variables not having differed significantly between the MSRD and non-MSRD groups in the corresponding univariate analyses. Sex was included fundamentally because female sex has frequently emerged as a significant risk factor in other studies; and acute rejection in the first yr because of its probably having given rise to the maintenance of larger doses of CNI, which in this study would make it a confounder.

Conversely, the multivariate analyses did not include immunosuppressive variables with respect to which the two groups had differed significantly in univariate analyses (with the exception of Tac vs. CsA in the last three multivariate analyses). Calcineurin inhibitors as a class were at first excluded because they are the root cause of post-transplant MSRD and were in any case initially administered to $95 \%$ of patients; while between-group differences in other immunosuppressants were regarded either as a consequence of concomitant differences in time since transplant and/or calcineurin inhibitor dosage or as a result of changes in therapy because of the detection of renal dysfunction (and hence not as risk factors). Thus, although the proportion of patients started post-operatively on azathioprine was larger in the MSRD than the non-MSRD group and the proportion started on mycophenolate smaller, we assume that this was partly because of the MSRD group having undergone transplantation at a slightly earlier date; and also, more decisively, to mycophenolate generally having been accompanied by lower doses of calcineurin inhibitors than azathioprine, as a consequence of which azathioprine-treated patients would be more likely to progress to MSRD (13). At the time of the study, the proportion of patients on azathioprine was smaller in the MSRD group, which we assume to have been because of the fact that for some yr, our centers, in view of our own experience (13) and the results of the IMPROVED study (14), have responded to renal dysfunction by switching patients from azathioprine to mycophenolate, with reduction or replacement of CNI. The greater prevalence of MTOR inhibitors in the MSRD group at study time is likewise attributable to their having been prescribed in response to the detection of renal dysfunction (15). By contrast, the inclusion of the variable Tac vs. CsA in the three final multivariate analyses is justified because in none of the 14 participating centers has it been standard practice to replace CsA with Tac in response to renal dysfunction.

Age at transplant has emerged as a risk factor in numerous studies $(1,5,11,16-19)$, even though the definition of renal dysfunction has varied considerably, from serum creatinine $>1.5 \mathrm{mg} / \mathrm{dL}$ (16) to GFR $<20 \mathrm{~mL} / \mathrm{min}$ per $1.73 \mathrm{~m}^{2}$ (19). Age is in fact expected to predict almost any GFR-based concept of renal dysfunction, since GFR in the general population aged $>30$ falls by about $1 \mathrm{~mL} / \mathrm{min}$ per $1.73 \mathrm{~m}^{2}$ per yr (4). Interestingly, age was not an independent predictor in two studies in which the criterion variable was end-stage renal disease (ESRD) (20, 21).

As noted above, female sex is also a risk factor for renal dysfunction that has been observed in many studies $(4,12,17,18)$, and which patients with HT "inherit" from the general population, where GFR is said to be around $8 \%$ less in women than in men (4). In the only study we know in which male sex rather than female sex emerged as a risk factor (16), this finding may have been related to the criterion for renal dysfunction having been very lax (serum creatinine $>1.5 \mathrm{mg} / \mathrm{dL}$ ) and/or to all patients having had normal pre-transplant serum creatinine values.

Time since transplant is likewise expected to increase the risk of renal dysfunction, partly because it contributes to age, and partly because it implies longer exposure to CNI for patients taking these drugs.

Another known risk factor for CKD - and for post-operative death (22) - is post-operative acute kidney injury (AKI), especially if renal replacement therapy is required for its reversal $(5,18)$. Whereas recovery from properly treated AKI is generally virtually total among non-transplant patients, it is hindered in transplant patients by immunosuppressive medication and by any functional underperformance by the transplanted organ (23). Post-operative AKI was not considered as such in the present study, but will no doubt have contributed to the influence of serum creatinine concentration $30 \mathrm{~d}$ 
post-HT, which must also reflect the reported influence of a sharp decline in renal function in the first three months after transplant (24). The emergence of pre-transplant serum creatinine as a risk factor independent of $30 \mathrm{~d}$ post-HT creatinine may also be due in part to the influence of the former on the incidence of post-operative AKI $(22,25)$.

Although the calcineurin inhibitors Tac and CsA were not found to differ in their renal effects in two short-term (12-18-month) multicenter studies in which 314 patients were randomized to one or the other $(26,27)$, there is increasing evidence that Tac not only improves blood lipid profile, but is also less renoaggressive than CsA following $\mathrm{HT}$, at least as regards stabilization or improvement of GFR and serum creatinine $(5,12,28-31)$. This conclusion appears to be supported by the results of the present study (in which the mean time since transplant was $6.7 \mathrm{yr}$ ): Tac was used by a greater proportion of nonMSRD than patients with MSRD both post-operatively and at the time of the study, and CsA by larger proportions of patients with MSRD than non-MSRD patients (Table 3); and Tac versus CsA at study time emerged as a significant predictor in the multivariate analyses in which it was included. Although it is not clear why Tac versus CsA at the institution of immunosuppression was not a significant predictor, this negative result may derive from Tac originally having been prescribed mainly to female patients - who are more prone to renal dysfunction - on account of the hirsutism associated with CsA (results not shown).

The above results must of course be considered with caution owing to the cross-sectional nature of this study. In particular, it is difficult to judge the extent to which they may have been affected by selective survival; for example, selective survival may have led to such acknowledged risk factors as diabetes, hypertension, and hyperlipidemia not emerging as such in this study. Also, although a distinction was made between post-operative immunosuppression and immunosuppression at the time of the study, no attempt was made to take into account the time point at which conversion from CsA to Tac may have taken place, changes in other reno-relevant medication (immunosuppressives, antihypertensives, etc.), or drug dosages or serum levels (though it is unlikely that generally recommended dosages can have been departed from). Nevertheless, none of the positive findings clashes with the recent literature in the field, and the lesser nephrotoxic risk of Tac at the time of the study emerged in spite of the above-noted greater prevalence of immunosuppression with Tac among female patients, who are more likely to progress to MSRD.

In conclusion, in this cross-sectional study of 1062 patients with HT, female sex, age, and serum creatinine at transplant, serum creatinine a month after transplant, time since transplant, and current use of CsA rather than Tac all emerged as statistically independent predictors of K/DOQI chronic kidney disease of stage 3 or worse. These findings should be tested further in prospective longitudinal trials.

\section{Authorship}

Designed study: Juan F. Delgado and MG Crespo. Performed study and collected data: J. F. Delgado, M. G. Crespo-Leiro, Miguel A. Gómez-Sánchez, M. J. Paniagua, F. González, J. A. Vázquez, J. Fernández, D. Pascual, L. Almenar, L. Martínez, B. Díaz, E. Roig, J. Segovia, J. M. Arizón, I. Garrido, T. Blasco, J. López, V. Brossa and N. Manito. Analyzed data: Javier Muñiz. Wrote the paper: Juan F. Delgado.

\section{Funding sources}

Astellas Pharma (unrestricted grant), and the Spanish Ministry of Health and Consumer Affairs through the Carlos III Institute Cardiovascular Research Networks REDINSCOR (JFD, LA, LM-D, ER, JS, and IG) and RECAVA (MC-L, MP and JM).

\section{Acknowledgements}

We thank Ian-Charles Coleman for helpful criticism and for the English version of this article. This research was supported by an unrestricted grant from Astellas Pharma and by the Spanish Ministry of Health and Consumer Affairs through the Carlos III Institute Cardiovascular Research Networks REDINSCOR (JFD, LA, LM-D, ER, JS and IG) and RECAVA (MC-L, MP and JM).

The Abstract was presented at the International Society for Heart and Lung Transplantation 29th Annual Meeting and Scientific Sessions, Paris (France), April 22-25th, 2009. 


\section{Appendix}

The following institutions and researchers participated in the CAPRI study (listed in descending order of the number of patients enrolled).

Complejo Hospitalario Universitario de A Coruña: Marisa Crespo Leiro, María J. Paniagua, Zulaika Grille, Carmen Naya, Victoria Prado.

Hospital Universitario 12 de Octubre, Madrid: Juan F. Delgado, Miguel Angel Gómez Sánchez, Pilar Escribano, Nuria Ochoa, Marta Paradina y María Vicente.

Hospital Universitario Marqués de Valdecilla, Santander: Francisco González- Vílchez, Jose A. Vázquez de Prada, Tamara García-Camarero, Miguel Llano.

Hospital General Universitario Gregorio Marañón, Madrid: Juan Fernández-Yáñez, David Pascual Hernández, Jesús Palomo Álvarez.

Hospital Universitario La Fe, Valencia: Luis Almenar, Luis Martínez-Dolz.

Hospital Universitario Central de Asturias, Oviedo: Beatriz Díaz-Molina, Jose Luis Rodríguez Lambert.

Hospital Clínic i Provincial, Barcelona: Eulalia Roig, Félix Pérez Villa.

Clínica Puerta de Hierro, Madrid: Luis Alonso-Pulpón, Javier Segovia, Manuel Gómez Bueno.

Hospital Universitario Reina Sofía, Córdoba: Jose M. Arizón, Juan Carlos Castillo Domínguez, Amador López Granados.

Hospital Universitario Virgen de la Arrixaca, Murcia: Iris P. Garrido, Domingo A. Pascual Figal.

Hospital Universitario Miguel Servet, Zaragoza: Teresa Blasco Peiró, Maria Luisa Sanz Julve.

Hospital Clínico Universitario, Valladolid: Javier López Díaz, Luis de la Fuente Galán. Hospital de la Santa Creu i Sant Pau, Barcelona: Vicens Brossa, Sonia Mirabet Pérez. Hospital Universitario de Bellvitge, Barcelona: Nicolás Manito Lorite.

Instituto de Ciencias de la Salud, Universidad de A Coruña: Javier Muñiz García.

\section{References}

1 Taylor DO, Edwards LB, Aurora P et al. Registry of the International Society for Heart and Lung Transplantation: Twenty-fifth official adult heart transplant report - 2008. J Heart Lung Transplant 2008: 27: 943. Data available at http://www.ishlt.org/registries/.

2 Myers BD, Ross J, Newton L, Luetscher J, Perlroth M. Cyclosporine-associated chronic nephropathy. N Engl J Med 1984: 311: 699.

3 Bloom RD, Doyle AM. Kidney disease after heart and lung transplantation. Am J Transplant 2006: 6: 671.

4 National Kidney Foundation Kidney Disease Outcome Quality Initiative Advisory Board. K/DOQI clinical practice guidelines for chronic kidney disease: evaluation, classification, and stratification. Am J Kidney Dis 2002: 39(Suppl. 2): S1.

5 Ojo AO, Held PJ, Port FK et al. Chronic renal failure after transplantation of a nonrenal organ. N Engl J Med 2003: 349: 931.

6 Alam A, Badonvinac K, Ivis F, Trpeski L, Cantarovich M. The outcome of heart transplant recipients following the development of end-stage renal disease: analysis of the Canadian Organ Replacement Register (CORR). Am J Transplant 2007: 7: 461.

7 Crespo-Leiro MG, Delgado JF, Paniagua MJ et al. Prevalence and severity of renal dysfunction among 1059 heart transplant patients according to criteria based on serum creatinine and estimated glomerular filtration rate: a cross-sectional study. J Heart Lung Transplant 2009: 28(2S): S263 (abstract).

8 Magee $\mathrm{C}$, Pascual M. The growing problem of chronic renal failure after transplantation of a nonrenal organ. $\mathrm{N}$ Engl J Med 2003: 349: 994.

9 Esposito C, Semeraro L, Bellotti N et al. Risk factors for chronic renal dysfunction in cardiac allograft recipients. Nephron 2000: 84: 21.

10 Wilkinson AH, Cohen DJ. Renal failure in the recipients of nonrenal solid organ transplants. J Am Soc Nephrol 1999: 10: 1136.

11 Hsu R-B, Chen RJ, Lin C-H et al. Renal dysfunction after heart transplantation: incidence, prognosis and risk factors. J Formos Med Assoc 2005: 104: 482.

12 Lewis GD, Frankel DS, Brown RN et al. Predictors of early development of chronic kidney disease following heart transplantation. J Heart Lung Transplant 2007: 26(2S): S137 (abstract).

13 Sánchez V, Delgado JF, Morales JM et al. Chronic cyclosporine-induced nephrotoxicity in heart transplant patients: long-term benefits of treatment with mycophenolate mofetil and low-dose cyclosporine. Transplant Proc 2004: 36: 2823.

14 Angermann CE, Stork S, Costard-Jäckle A et al. Reduction of cyclosporine after introduction of mycophenolate mofetil improves chronic renal dysfunction in heart transplant recipients - The IMPROVED multi-centre study. Eur Heart J 2004: 25: 1626.

15 Delgado J, Crespo MG, Manito N et al. Sirolimus (SRL) in heart transplantation: preliminary results of a multicenter registry in Spain (Rapacor Study). Am J Transplant 2005: 5(S11): 172 (abstract).

16 Garrido IP, Crespo-Leiro MG, Paniagua MJ et al. Independent predictors of renal dysfunction after heart transplantation in patients with normal pretransplant renal function. J Heart Lung Transplant 2005: 24: 1226. 
17 Diez-Ojea B, Gago-González E, Díaz-Corte C et al. Study of the renal function in nonrenal organ transplantation. Transplant Proc 2006: 38: 2985.

18 Hamour IM, Omar F, Lyster HS, Palmer A, Banner NR. Chronic kidney disease after heart transplantation. Nephrol Dial Transplant 2009; 24: 1655.

19 Lindelow B, Bergh C-H, Herlitz H, Waagstein F. Predictors and evolution of renal function during 9 years following heart transplantation. J Am Soc Nephrol 2000: 11: 951.

20 Satchithananda DK, Parameshwar J, Sharples L et al. The incidence of end-stage renal failure in 17 years of heart transplantation: a single center experience. J Heart Lung Transplant 2002: 21: 651.

21 Rubel JR, Milford EL, McKay DB, Jarcho JA. Renal insufficiency and end-stage renal disease in the heart transplant population. J Heart Lung Transplant 2004: 23: 289.

22 Boyle JM, Moualla S, Arrigain S et al. Risks and outcomes of acute kidney injury requiring dialysis after cardiac transplantation. Am J Kidney Dis 2006: 48: 787.

23 Bloom RD, Reese PP. Chronic kidney disease after nonrenal solid-organ transplantation. J Am Soc Nephrol 2007: 18: 3031 .

24 Cantarovich M, Hirsh A, Alam A et al. The clinical impact of an early decline in kidney function in patients following heart transplantation. Am J Transplant 2009: 9: 348 .

25 Odim J, Wheat J, Laks $\mathrm{H}$ et al. Peri-operative renal function and outcome after orthotopic heart transplantation. $\mathrm{J}$ Heart Lung Transplant 2006: 25: 162.

26 Taylor DO, Barr ML, Radovancevic B et al. A randomized, multicenter comparison of tacrolimus and cyclosporine immunosuppressive regimens in cardiac transplantation: decreased hyperlipidemia and hypertension with tacrolimus. J Heart Lung Transplant 1999: 18: 336.

27 Grimm M, Rinaldi M, Yonan NA et al. Superior prevention of acute rejection by tacrolimus vs. cyclosporine in heart transplant recipients - a large European trial. Am J Transplant 2006: 6: 1387.

28 Israni A, Brozena S, Pankewycz O, Grossman R, Bloom R. Conversion to tacrolimus for the treatment of cyclosporine-associated nephrotoxicity in heart transplant patients. Am J Kidney Dis 2002: 39: E16.

29 Garlicki M, Czub P, ŁAbuś K, Ehrlich MP, Rdzanek H. Conversion from cyclosporine to tacrolimus improves renal function and lipid profile after cardiac transplantation. Ann Transplant 2006: 11: 24.

30 Kobashigawa JA, Miller LW, Russell SD et al. Tacrolimus with mycophenolate mofetil (MMF) or sirolimus vs. cyclosporine with MMF in cardiac transplant patients: 1-year report. Am J Transplant 2006: 6: 1377.

31 Kobashigawa JA, Patel J, Furukawa $\mathrm{H}$ et al. Five-year results of a randomized, single-center study of tacrolimus vs microemulsion cyclosporine in heart transplant patients. J Heart Lung Transplant 2006: 25: 434. 